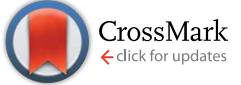

Cite this: J. Mater. Chem. A, 2016, 4 , 16136

Received 16th August 2016

Accepted 14th September 2016

DOI: 10.1039/c6ta07023j

www.rsc.org/MaterialsA

\section{Controlling additive behavior to reveal an alternative morphology formation mechanism in polymer : fullerene bulk-heterojunctions $\uparrow$}

\author{
Nusret S. Güldal, ${ }^{* a}$ Marvin Berlinghof, ${ }^{b}$ Thaer Kassar, ${ }^{b}$ Xiaoyan Du, ${ }^{c}$ Xuechen Jiao, ${ }^{d}$ \\ Markus Meyer, ${ }^{c}$ Tayebeh Ameri, ${ }^{a}$ Andres Osvet, ${ }^{a}$ Ning Li, ${ }^{a}$ Giovanni Li Destri, ${ }^{\text {e }}$ \\ Rainer H. Fink, ${ }^{c}$ Harald Ade, ${ }^{d}$ Tobias Unruh ${ }^{b}$ and Christoph J. Brabec ${ }^{a}$
}

One of the most employed morphology optimization methods to design better performing organic photovoltaic devices is ink formulation engineering with additives. In this work, by employing a suboptimal host solvent mixture and 1,8-diiodooctane (DIO) as a very optimal solvent for both components in poly-thieno[3,2b]thiophene-diketopyrrolopyrrole-co-thiophene (DPP-TT-T)-based bulkheterojunctions $(\mathrm{BHJ})$, an alternative, previously unknown mechanism of additive behavior on $\mathrm{BHJ}$ microstructure formation is presented. In situ characterization methods involving grazing incidence $X$-ray diffraction, white-light reflectometry, laser light scattering and photoluminescence during film drying reveal that the microstructure formation under the influence of DIO is led towards thermodynamic equilibrium during host solvent drying, and the kinetics of morphology formation (i.e. polymer crystallization, fullerene aggregation...) are controlled dominantly by the additive during its evaporation. Ex situ X-ray-based characterization methods, such as scanning transmission X-ray microspectroscopy (STXM) and resonant soft X-ray scattering (R-SoXS), additionally reveal that the microstructure of dried films favors smaller domain sizes with purer domains, smaller fullerene aggregates, bimodal polymer crystallization relative to the substrate and more face-on molecular orientation relative to the donor/acceptor interface, which at the end lead to better performing devices with power conversion efficiencies ranging from $1.25 \%$ to $4.68 \%$.

\section{Introduction}

In the last two decades, organic solar cells (OSCs) have attracted a tremendous amount of interest as an alternative to conventional inorganic systems due to their easy and scalable production. Organic photoactive materials can be printed from optimized inks on large-scale flexible substrates with different roll-to-roll techniques, such as slot-die coating and gravure coating. ${ }^{1}$ As the state-of-the-art power conversion efficiency (PCE) of single-junction OPVs has exceeded 10\%, these advantages make OPVs great candidates for sustainable energy conversion. ${ }^{2}$

${ }^{a}$ Materials for Energy Technology and Electronics, Friedrich-Alexander University Erlangen-Nürnberg, Martensstrasse 7, 91058, Erlangen, Germany. E-mail: nusret.s. guldal@fau.de

${ }^{b}$ Chair for Crystallography and Structural Physics, Friedrich-Alexander University Erlangen-Nürnberg, Staudtstrasse 3, 91058, Erlangen, Germany

'Physikalische Chemie, Friedrich-Alexander University Erlangen-Nürnberg, Egerlandstrasse 3, 91058, Erlangen, Germany

${ }^{d}$ Department of Physics, North Carolina State University, Raleigh, NC, 27695, USA ${ }^{e}$ European Synchrotron Radiation Facility, 71 Avenue des Martyrs, 38000, Grenoble, France

$\dagger$ Electronic supplementary information (ESI) available. See DOI: $10.1039 /$ c6ta07023j
An optimized bulk-heterojunction active layer has a phase separated, yet finely mixed morphology, which ensures the most efficient exciton dissociation and charge transport within the system. ${ }^{3}$ Recently, there are major scientific efforts to understand and manipulate microstructural effects on the device performance. ${ }^{\mathbf{4 - 1 2}}$ Several methods have been developed to influence the morphology and consequently the performance of OSCs, such as solution optimization, thermal annealing and solvent annealing. ${ }^{4-8}$ Among all these methods, employing low volatile additives has become a well-accepted microstructure manipulation method, especially to the systems for which postprocessing treatments have been proven ineffective..$^{5,9-12}$

Two guidelines followed when selecting an effective additive are: (1) the additives should be non-solvents or worse solvents than host solvents for one component (generally for the donor material), and (2) the additive should have lower volatility than the host solvent. ${ }^{13}$ Although 1-chloronaphthalene $(\mathrm{CN})$ is an exception among all additives since it has been reported to provide good solubility for both components, it is still a worse solvent for the donor material than the host solvents themselves; hence the working principle of all additives, including CN, 1,8-diiodooctane (DIO) and alkanedithiols, has been attributed to these conditions.,.$^{\mathbf{9} 10,13-15}$ Nevertheless, utilizing 
such additives is detrimental for all-solution printed flexible OPVs since most of the additives stay in the film due to their low volatility, affecting reproducibility and performance detrimentally if not properly removed. ${ }^{16,17} \mathrm{Few}$ removal processes have been proposed for residual solvents and additives, like vacuum processes and washing with highly volatile alcohols. ${ }^{16-19}$ In spite of the effectiveness of these methods, none of them is applicable to large-scale coating methods. Thus, it is extremely important to understand the precise mechanism of additives on nanostructure formation in close coordination with solution thermodynamics, thin film drying kinetics and the device performance, in order to design better ink formulations, eventually without additives.

Over the past years, there has been a great amount of efforts to gain nanoscale structural information about additive effects on solution-processed polymer: fullerene blend thin films, mainly after processing (ex situ measurements). ${ }^{20-23}$ However, it was necessary to observe film formation during drying in order to gain better insight into the role of additives in microstructure formation, thus controlling the morphology with better functionality. Stand-alone or combined measurement method studies were conducted on microstructure evolution during drying of spin-coated, doctor bladed or roll-to-roll coated polymer : fullerene bulk heterojunction (BHJ) films. ${ }^{24-37}$ Very few of these in situ studies have focused on additive effects. The common outcome of these studies is that whether the selected additive is a good solvent or not, additives are still worse solvents for the donor material than the host solvents. Hence, although the end morphology (crystal size, domain size, phase separation...) might be different due to the interaction of blend components with different additives depending on the solubility, the evolution of morphology formation is similar: additives reduce the quality of the host solvents by accelerating the initiation of polymer aggregation/crystallization, prolong the drying and provide more time for crystallization, i.e. polymer ordering. ${ }^{32-37}$ Although these studies provided invaluable information on the matter, there are very few examples of additives with different solubility behaviors; hence the current understanding on the relationship between solution thermodynamics and drying kinetics of such thin films is not detailed enough to build a roadmap for producing better ink formulations for the purpose of upscaling.

Recently, we demonstrated a portable and atmospherecontrolled in situ measurement setup for polymer : fullerene thin films based on poly-thieno[3,2b]thiophene-diketopyrrolopyrrole-co-thiophene (DPP-TT-T) and [6,6]-phenyl- $\mathrm{C}_{61}$-butyric acid methyl ester (PCBM) (Scheme S1†), and observed the drying kinetics and microstructure formation with three measurement methods, namely white-light reflectometry (WLR), laser light scattering (LS) and photoluminescence (PL), simultaneously. ${ }^{38}$ In this manuscript, we extend the study on DPP-TT-T : PCBM by utilizing DIO as a better solvent for both components than the host solvents in order to visualize the precise function of an additive in an ink formulation and to describe an alternative morphology formation mechanism for polymer : fullerene bulk-heterojunctions. For that matter, we use our in situ characterization setup to examine the drying kinetics and morphology evolution. Our in situ studies, together with various $e x$ situ X-ray and optical characterization methods, revealed that DIO, as a much better solvent for both components than the host solvents, leads the evolution of morphology formation towards thermodynamic equilibrium (i.e. none of the kinetic mechanisms takes place during host solvent drying). Our study shows that the functioning mechanism of additives depends heavily on the host solvent quality for the selected blend, and thus an improved device performance can also be achieved even if the morphology formation occurs solely during additive drying. Hence, our study emphasizes that the selection criteria for effective additives for any bulk-heterojunction system described earlier should not be taken as fixed rules, and the overall solution thermodynamics must be extensively investigated in close relation with microstructure formation kinetics.

\section{Results and discussion}

\section{Device performance}

DPP-TT-T : PCBM solutions were prepared with a solvent mixture of $o$-xylene and mesitylene at $60{ }^{\circ} \mathrm{C}$. Solar cells were fabricated in inverted structure by doctor blading at $60^{\circ} \mathrm{C}$ under air (Fig. 1a). The thermal evaporation of electrodes for additivefree cells was performed at $2 \times 10^{-6}$ mbar pressure while additive-containing cells had two types of vacuum pressure. One evaporation batch with 2 vol\% DIO was conducted at $5 \times$ $10^{-5}$ mbar without letting the additive out of the active layer completely. The rest of the additive-containing samples (labelled as evacuation (Eva)) were vacuumed for 30 minutes at the minimum pressure the evaporation chamber could reach, which was $2 \times 10^{-6}$ mbar, before the electrodes were thermally evaporated. As seen from the current density vs. voltage curve and device characteristics in Fig. $1 \mathrm{~b}$ and Table $\mathrm{S} 1, \dagger$ respectively, the device performance was exceptionally poor in the absence of DIO. With addition of DIO, the gain in power conversion efficiency (PCE) was an effect of both the short circuit current density $\left(J_{\mathrm{SC}}\right)$ and fill factor $(\mathrm{FF})$. In fact, $J_{\mathrm{SC}}$ and $\mathrm{FF}$ showed an increase already with $1 \mathrm{vol} \%$ DIO. The sample labelled as $1 \mathrm{vol} \%$ DIO + Eva reached a PCE value of $1.89 \%$, while the sample with 2 vol\% DIO + Eva showed a $J_{\mathrm{sC}}$ and PCE of $13.89 \mathrm{~mA} \mathrm{~cm}{ }^{-2}$ and $4.68 \%$, respectively. With 4 vol\% DIO, however, $J_{\mathrm{SC}}$ dropped to $8.48 \mathrm{~mA} \mathrm{~cm}^{-2}$, subsequently lowering the PCE to $3.12 \%$.

The evidence of residual additive in the processed layers, which causes detrimental effects on the cell performance, was reported earlier by several groups. It has been proven that the applied pressure and the evacuation time under the selected pressure are two crucial factors to remove residual additives from the bulk-heterojunction layers successfully. ${ }^{16,39}$ In order to complement the literature and to prove the existence of residual DIO after $10^{-5}$ mbar pressure for the sake of our ex situ measurements, a device with 2 vol\% DIO under $5 \times 10^{-5}$ mbar thermal evaporation was built and compared. Depending on the breadth of the effect that the residual additive causes in the blend, the performance difference between the properly evacuated sample and the sample with residual additive might differ. $^{16,39}$ In our case, residual DIO in the system causes 
(a)

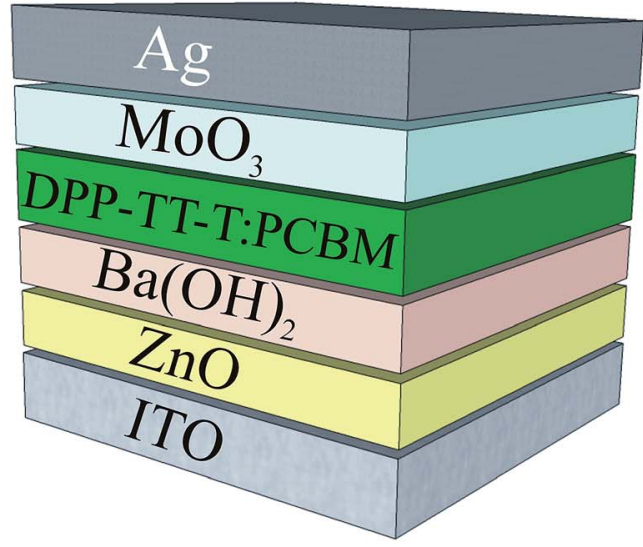

(b)

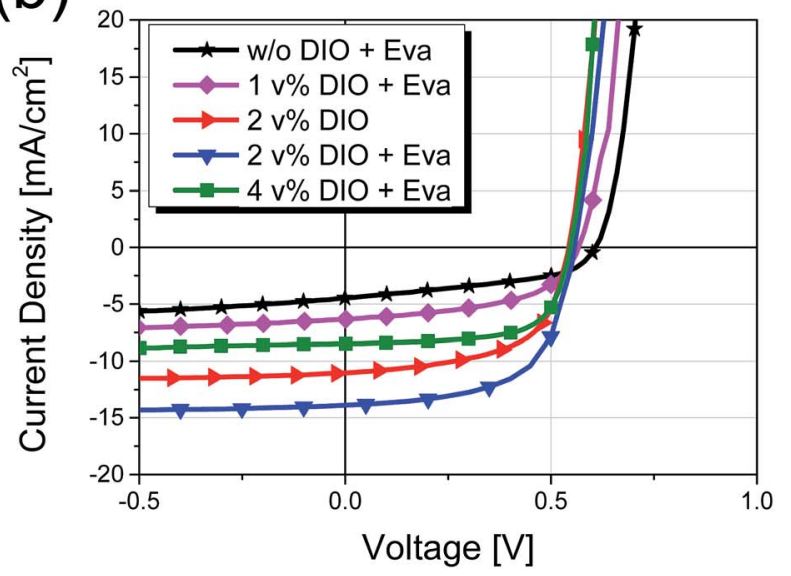

Fig. 1 The structure (a) and the performance (b) of organic solar cells are represented. Solar cells are measured under illumination of an AM 1.5 solar simulator. Additive-free, 1 and 4 vol\% DIO samples experienced a full vacuum procedure before top electrode evaporation. The effect of residual DIO was tested only on the 2 vol\% DIO sample.

a decrease in almost all parameters, the most in $J_{\mathrm{SC}}$ from 13.89 to $11.03 \mathrm{~mA} \mathrm{~cm}^{-2}$, leading to a PCE of $3.53 \%$.

\section{Ex situ characterizations}

Ex situ characterization methods were applied to visualize the structural differences and correlate these structural factors with device performances. Since the devices showed the best performance with 2 vol\% DIO, ex situ characterization measurements were applied only to the layers with 2 vol\% DIO, and the results were compared with additive-free layers.

Steady-state UV-Vis absorption \& PL measurements. UV-Vis absorption measurements revealed that the two peaks at 740 and $800 \mathrm{~nm}$ in Fig. 2a are characteristic peaks originating from DPP-TT-T, which are correlated with the structural ordering. ${ }^{\mathbf{4 0}}$ The $740 \mathrm{~nm}$ peak was reduced as PCBM was added, indicating that PCBM disturbed the local ordering to a certain extent. Adding 2 vol\% DIO suppressed the $740 \mathrm{~nm}$ peak even further, which recovered after the evacuation process. Furthermore, PL measurements (Fig. 2b) show that in the additive-free sample, PCBM quenched the DPP-TT-T peak at $920 \mathrm{~nm}$, while the characteristic PCBM aggregate peak emerged at $720 \mathrm{~nm} .{ }^{41}$ With DIO, the DPP-TT-T peak intensity increased, and PCBM peak blue-shifted and weakened. After evaporation of the additive, the DPP-TT-T peak broadened and lost intensity, while the PCBM peak recovered the intensity with an additional shoulder appearing at $680 \mathrm{~nm}$. The reason behind this specific PL behavior of a luminescent polymer in the presence of an additive (1) and after evaporation of the additive (2) is as follows: (1) DIO increases the phase separation between the polymer and the fullerene, leading to changes in the local polymer : fullerene composition, thus increasing the PL signal. (2) Evaporation of DIO leads to better ordering of the polymer, as well as remixing of the quencher and the polymer, causing slightly lower PL signals. It is further deduced that the presence of an additive (DIO) in general increases phase separation; hence the DPP-TTT PL signal is higher than the PL signal of the additive-free film.
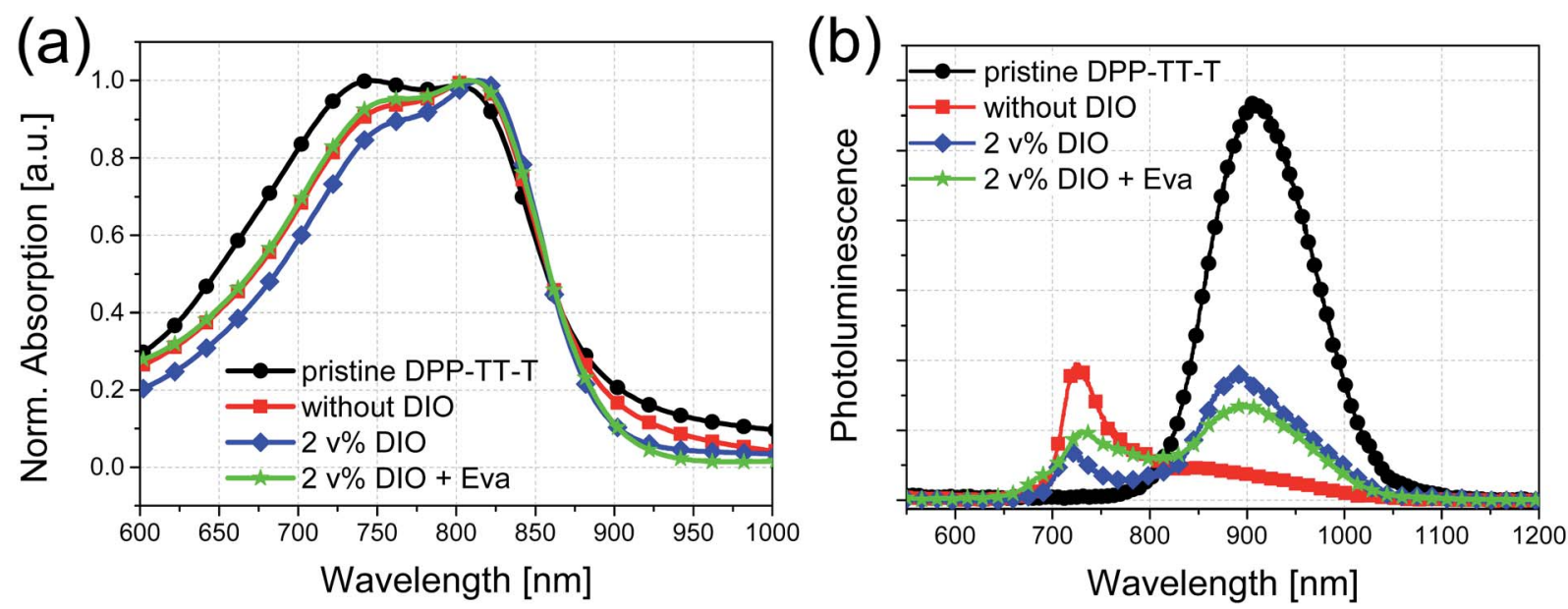

Fig. 2 (a) Normalized absorption spectra and (b) optical density corrected PL spectra of pristine DPP-TT-T and blends with and without additive. 
In order to further correlate the steady-state PL measurements and device performances with the morphological properties of the processed films, the following surface and bulk characterization methods were applied: AFM, BF-TEM, STXM, NEXAFS, R-SoXS and GIXD.

\section{Domain characteristics \& nature of crystals}

In order to visualize the nature of the phases, microscopic probes like AFM, BF-TEM and STXM were employed (Fig. 3). AFM gives sensitive information on the surface morphology, yet not capable of imaging the nanoscale morphology in the bulk. On the other hand, BF-TEM and STXM are more sensitive to the bulk morphology. The contrast in STXM is achieved through variations in the near-edge X-ray absorption fine structure (NEXAFS), thus providing information on the chemical and phase composition.

AFM micrographs indicate that DPP-TT-T : PCBM without DIO had showed large features in the range of $350 \mathrm{~nm}$. This was confirmed by BF-TEM and STXM, where fullerene aggregates (dark regions) with a size of $350 \mathrm{~nm}$ were observed as well. Upon addition of DIO, the film gained more homogeneity and smoothness, and BF-TEM and STXM showed smaller fullerene aggregates throughout the bulk. When DIO was completely removed, the film regained its domain features with fullerene aggregates in the order of $100 \mathrm{~nm}$. Regardless of the PCBM aggregates, the film contained smaller domains in the mixed phase after evaporation (Fig. S1 $\dagger$ ). In fact, the additive-free sample had domains in the order of $50 \mathrm{~nm}$, while DIO-containing samples had smaller domains with approximately $30 \mathrm{~nm}$ of size. It should be noted that the term 'mixed phase' is used here to describe the parts of the film without any fullerene aggregates, and thus includes crystalline and amorphous regions.

Further characterization of the domain spacing and relative average domain purity in binary blends was conducted with
R-SoXS, which is sensitive to both the crystalline and amorphous fraction of active layers. Measurements were performed at a resonant energy of $283.8 \mathrm{eV}$, in order to provide a high material contrast between DPP-TT-T-rich domains and PCBMrich domains and to reduce mass-thickness variation effects caused by the vacuum contrast (for further details, see ESI $\dagger$ ). Lorentz-corrected R-SoXS scattering data $\left(I_{\text {avg }} q^{2}\right.$, circular $)$ are plotted against the scattering vector $q$ (Fig. 4a). The domain spacing is represented by the characteristic median length scale, which is a conversion of the $q$ point equal to the half of the integrated scattering intensity (ISI, eqn (S1) $\dagger$ ) by $2 \pi$ (eqn $(\mathrm{S} 2) \dagger)$. The characteristic median length scale for additive-free, with DIO and DIO + Eva samples was calculated to be 56, 29 and $29 \mathrm{~nm}$, respectively, which match very well with the domains measured within the mixed phase by BF-TEM. These results strongly indicate that the addition of DIO decreased the domain size to almost half of the additive-free sample, which subsequently increased the interfacial area required for the efficient exciton dissociation, and thus increased $J_{\mathrm{SC}}$.

In addition to domain spacing, the ratios of integrated scattering intensities (ISI) between the samples can be used to analyze relative average domain purity, where stronger ISI indicates higher domain purity. ${ }^{42}$ The ISI ratio of samples without and with DIO was calculated to be $1: 1.67$, showing that DIO enforces purer domains, which consequently explains the better FF observed in samples with DIO. On the other hand, the ISI ratio of samples with DIO + Eva and with DIO was $1: 1.36$, which indicates a slight loss of domain purity after the evaporation of DIO. Since PCBM is more soluble in DIO than DPP-TTT, DIO kept more PCBM separate and mobile from the rest of the mixed phase, as explained earlier with STXM and BF-TEM measurements. After the evaporation of DIO, PCBM slightly intermixed within the domains, thus reducing the purity compared to the DIO sample without evaporation. Although the intermixing behavior supports the increase in $J_{\mathrm{SC}}$, the slight

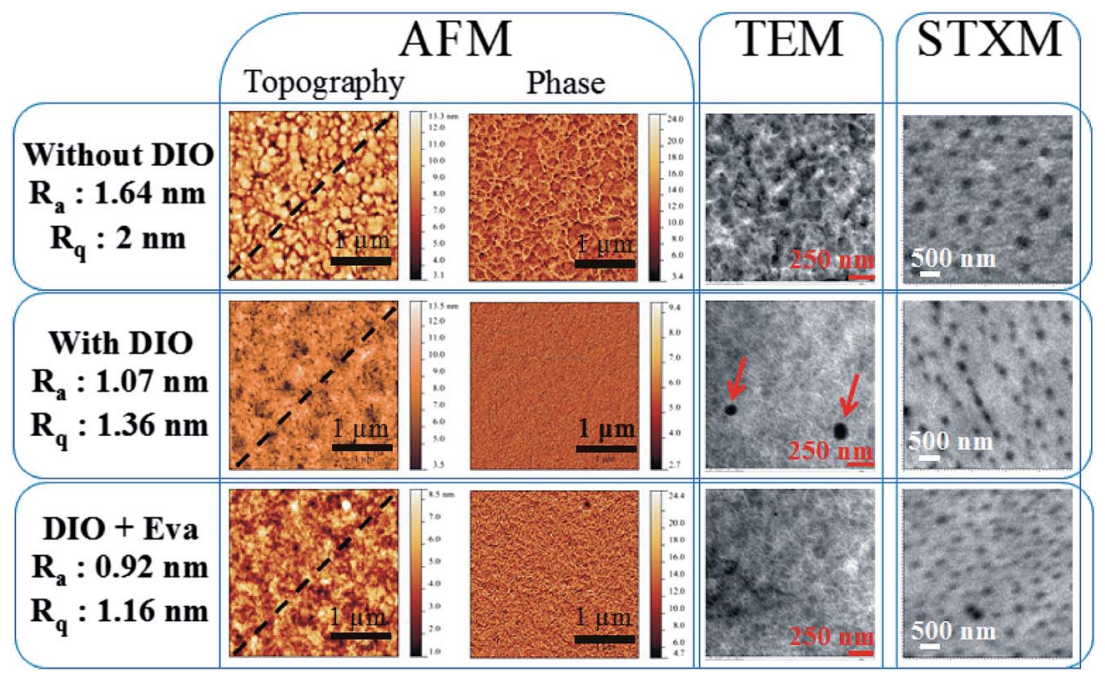

Fig. 3 AFM topography and phase images (left), BR-TEM (middle) and STXM ( $h \nu=284.5 \mathrm{eV}$ ) pictures (right) are shown. Black dashed lines at AFM topography images indicate the lines where the roughness calculations were done. The corresponding roughness values are given below the name tags. The arrows in BR-TEM pictures for the sample with DIO indicate the PCBM aggregates. 
(a)

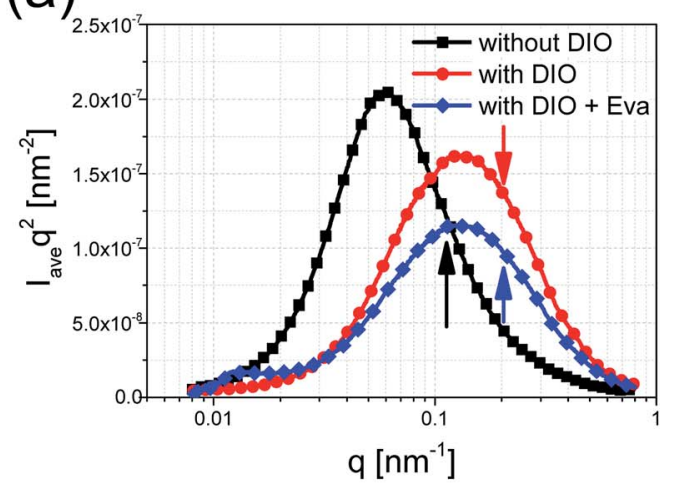

(b)

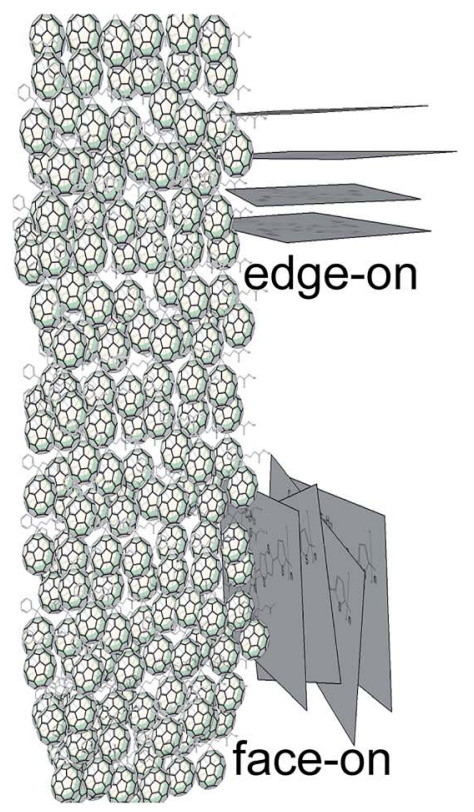

(c)

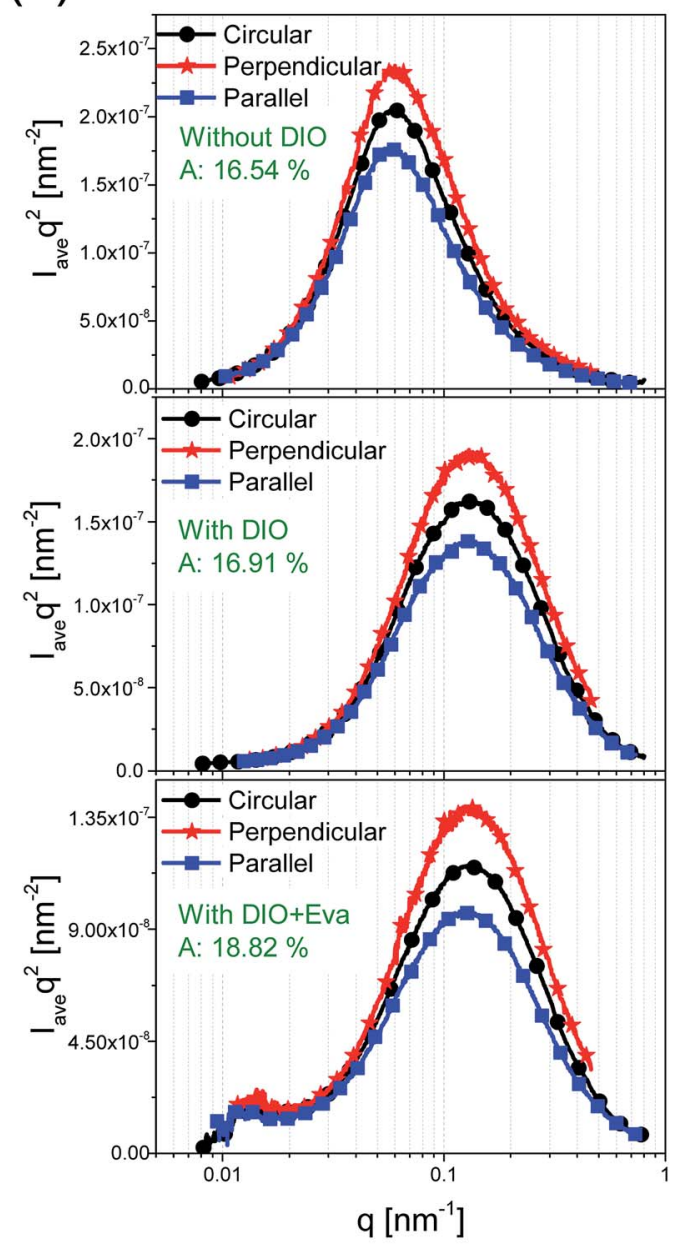

Fig. 4 Lorentz-corrected circular R-SoXS scattering profiles of additive-free DPP-TT-T : PCBM, with DIO and with DIO + Eva (a). The arrows in (a) indicate the $q$ values from which the median characteristic lengths were calculated, as explained more in detail in the main text. (b) is a schematic representation of the material orientation correlation between polymer-rich and fullerene-rich domains relative to the donoracceptor interface. (c) shows the Lorenz-corrected P-SoXS scattering profiles and sectors perpendicular and parallel to the X-ray polarization, for each sample. Calculated anisotropy ratios for each sample are given in the corresponding graphs, as $16.54,16.91$ and $18.82 \%$.

increase in FF after DIO evaporation despite the intermixing is attributed to the polymer crystallization and molecular orientation, which is explained later in the text.

In order to visualize the compositional changes within the mixed phase, NEXAFS was taken subsequent to the STXM measurements (Fig. 5 and S3†). In the NEXAFS spectra (Fig. 5), the characteristic peak intensities for PCBM $(284.5 \mathrm{eV})$ and DPPTT-T $(285.2 \mathrm{eV})$ show clear changes under different processing conditions. The experimental data were fitted using reference spectra of each component to attain local chemical composition variations (for further details on the analysis, see ESI, Fig. S3 and Table S2 $\uparrow$ ). The PCBM chemical composition was reduced from $63 \%$ to $57.6 \%$ in the presence of DIO. The evaporation step, however, increased the PCBM chemical composition from $57.6 \%$ to $60 \%$ in the mixed phase. These results indicate that PCBM within the mixed phase separated in the presence of DIO. Slight intermixing behavior from PCBM aggregates towards the mixed regions was observed after the evaporation of the additive, thus increasing the PCBM chemical composition. These results including the domain purity results from R-SoXS complement the steady-state PL signals of DPP-TT-T very strongly: DIO removed a fraction of PCBM from the mixed region, and thus led to an increase in the DPP-TT-T PL signal while the removal of DIO induced a slight intermixing of PCBM, reducing the DPP-TT-T PL signal.

The nature of DPP-TT-T crystals was analyzed with GIXD (Fig. 6a and b). DPP-TT-T is a semi-crystalline polymer, with edge-on orientation with $h 00$ Bragg peaks at $0.24,0.51$ and $0.75 \AA^{-1}$ at $q_{z}$ cuts. The 010 peak was not visible for the additivefree sample in either the $q_{z}$ or $q_{y}$ cut, probably due to the very low intensity. The PCBM peak was observed at $1.47 \AA^{-1}$. On the other hand, the sample with DIO showed a complete amorphous behavior, while the PCBM peak was weakened and shifted to $1.55 \AA^{-1}$. After evaporation of DIO, the sample 


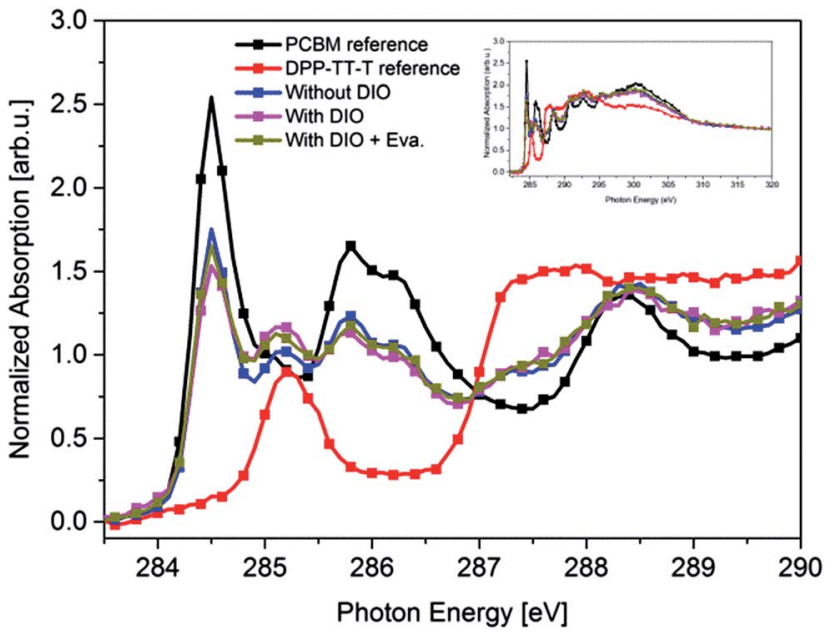

Fig. 5 Normalized NEXAFS spectra of thin films of additive-free DPPTT-T : PCBM, DPP-TT-T : PCBM with DIO and DPP-TT-T : PCBM with DIO after the removal of DIO (i.e. Eva). Reference spectra of PCBM and DPP-TT-T were used for spectral deconvolution to derive the chemical composition. The change in the relative characteristic peak intensity for PCBM and DPP-TT-T in the different blend films is clear. The inset shows spectra in an extended photon energy range.

recovered the edge-on orientation, and an additional broad [010] peak ( $\pi-\pi$ stacking) appeared at $1.97 \AA^{-1}$ in the $q_{z}$ direction, which is characteristic of face-on orientation. Compared to the additive-free sample, DIO-Eva samples showed bimodal crystallization preference, with both face-on and edge-on orientations. These results strongly suggest that DIO is a good solvent for DPP-TT-T. Previously, steady-state UV-Vis measurements revealed that DIO induced a diminished vibronic peak of DPPTT-T, which was interpreted as the disturbed local order. Interestingly, GIXD measurements showed that DPP-TT-T ordering was completely suppressed by DIO, and the evaporation of DIO was necessary to re-crystallize DPP-TT-T. In particular, the bimodal behavior of the crystals could be an additional source for FF enhancement, as the polymer ordering is a very dominant factor affecting the charge transport and interconnectivity within the bulk-heterojunction, thus improving the solar cell performance. ${ }^{43}$

The molecular orientation correlation between polymer-rich and fullerene-rich domains relative to the donor/acceptor interface was found to be well connected with the charge separation in several bulk-heterojunction systems. ${ }^{\mathbf{4 4 , 4 5}}$ In order to quantify the degree of molecular orientation correlation, a scattering anisotropy value is calculated from the polarized soft X-ray scattering (P-SoXS) profiles. P-SoXS profiles with horizontal X-ray polarization are plotted in Fig. 4c, in which the scattering perpendicular to the X-ray polarization showed a stronger signal than the scattering parallel to the X-ray polarization. The P-SoXS profiles are discussed in terms of the scattering anisotropy factor, which describes a complete face-on $(+1)$ or a complete edge-on $(-1)$ molecular orientation correlation between polymer-rich and fullerene-rich domains relative to the $\mathrm{D} / \mathrm{A}$ interface. With respect to this definition, the anisotropy ratio for the additive-free sample was calculated to be $16.54 \%$ (Fig. 4c). The sample with residual DIO experienced a slight increase in the ratio up to $16.91 \%$, while the removal of DIO enforced an evident increment to $18.82 \%$. This indicates that the removal of DIO enabled an enhanced face-on molecular orientation correlation between polymer-rich and fullerene-rich domains relative to the donor/acceptor interface.

\section{In situ characterizations}

In our previous work, we combined in situ WLR, PL, LS and GIXD measurements and observed the drying mechanism of additive-free DPP-TT-T : PCBM at $60{ }^{\circ} \mathrm{C}$ under an inert atmosphere. ${ }^{38}$ We were able to attain a complete picture on the interplay between the thermodynamic and kinetic processes, governing the drying. In particular from in situ PL measurements, we concluded that the microstructure formation of additive-free DPP-TT-T : PCBM was dominated by the solubility (a)

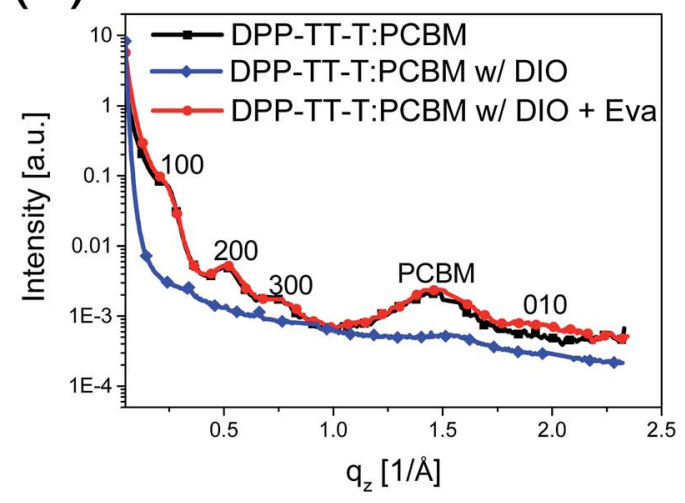

(b)

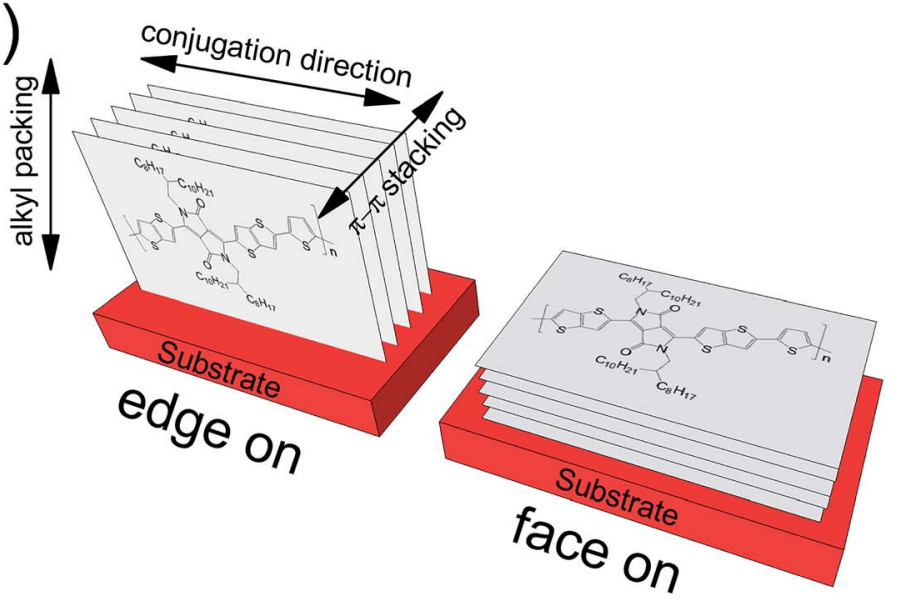

Fig. 6 (a) GIXD profiles of additive-free and additive containing samples in the out of plane direction. It should be noted that the GIXD profile of DPP-TT-Z : PCBM with DIO was obtained from in situ measurements, at a time point right after the host solvent completely evaporated, while other samples were measured ex situ. (b) Schematic of face-on and edge-on polymer ordering with respect to the substrate. 
limit of the polymer in $o$-xylene which caused a solid-liquid phase separation-induced gelation, hindering the crystal growth. Here, we extend our in situ study on DPP-TT-T : PCBM with different amounts of DIO, in order to observe the ternary solvent system effects on the microstructure evolution and to reveal an alternative additive functionality on the drying of a polymer : fullerene system.

Samples without additive and with 1, 2 and 4 vol\% DIO were bladed and dried at $60{ }^{\circ} \mathrm{C}$ in our in situ drying setup (Fig. S4 $\dagger$ ), where we measured WLR, LS and PL in parallel during drying. Thickness profiles for all samples (Fig. 7a) were calculated from the interference patterns of the reflectance spectra measured by WLR (see ESI for details, Fig. S9†). All samples dried at a constant rate predominantly, indicating evaporation- dominated drying. The additive-free sample dried around the $39^{\text {th }}$ second without any diffusion-dominated drying while DIOcontaining samples entered a slow drying regime due to the low vapor pressure of DIO. Nevertheless, DIO in the film did not affect the host solvent drying rate, as the drying curves for all samples including pristine DPP-TT-T films with and without additive followed an almost identical drying behavior until the host solvent completely evaporated (Fig. 7a and S5†).

In our previous work, we argued that LS of the DPP-TT$\mathrm{T}$ : PCBM sample without DIO did not originate from lateral or vertical phase separation as suggested earlier for similar systems, but rather from fullerene aggregates with a size in the order of the incident wavelength. ${ }^{36,38}$ The LS signal of the additive-free sample (Fig. 7b) showed a sharp increase right
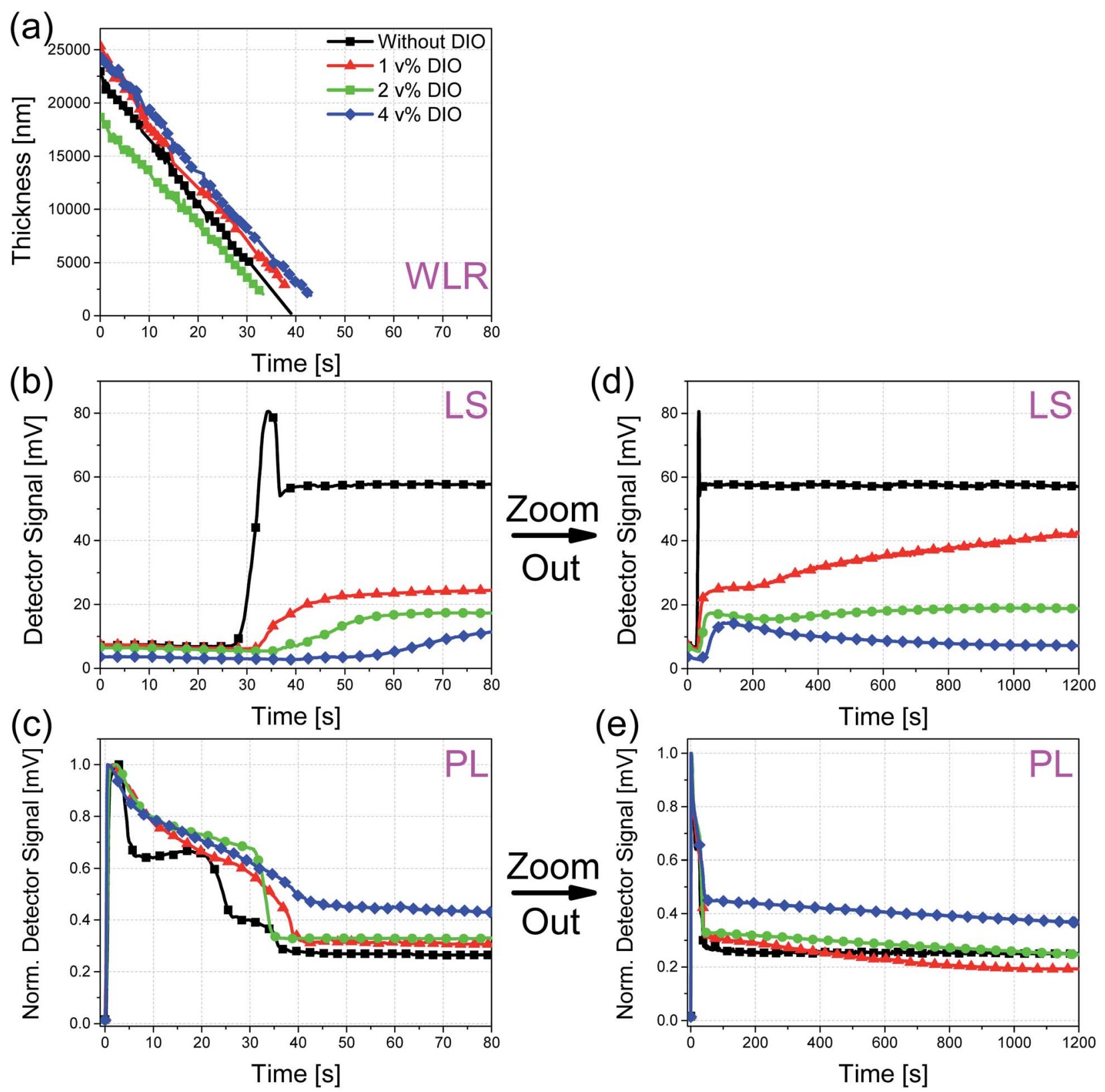

Fig. 7 Time evolution of DPP-TT-T : PCBM drying was measured by WLR (a), LS (b) \& (d), PL (c) \& (e). (b) and (c) are additionally 'zoomed out' (i.e. plotted over a longer time scale), which are presented in (d) and (e), respectively. 
before drying and stabilized quickly with the completion of drying. With 1 vol\% DIO, the signal started to increase slowly and only slightly, however it continued to increase until the $1000^{\text {th }}$ second of the drying (Fig. 7d). This was very well expected as DIO in the film kept PCBM soluble, and thus the size of the final aggregates stayed smaller than the aggregates observed in the additive-free sample, which are in excellent agreement with the trend observed by STXM and BF-TEM measurements. As the DIO amount in the wet film was increased, the time point of the first signal was delayed. Additionally, the intensity of the first signal reduced since more DIO kept more PCBM mobile, thus reducing the aggregate size.

In situ PL measurement of the additive-free DPP-TT-T : PCBM film showed very distinctive drying patterns (Fig. 7c), which were discussed previously in great detail. ${ }^{38}$ In particular, the region between $10^{\text {th }}$ and $20^{\text {th }}$ seconds of drying was attributed to the gelation of DPP-TT-T in $o$-xylene after reaching its solubility limit of $8 \mathrm{mg} \mathrm{mL}$. Hence the diffusion-assisted microstructure evolution was frozen for this period of time (i.e. indicated by the stable PL signal for a short period of time).$^{38}$ The additive suppressed this behavior; hence the plateau pattern in the PL signal vanished. Additionally, as the concentration of DIO was increased, the PL signal after the host solvent drying stayed higher.

In order to realize whether these effects on the PL signal originated due to the mobile PCBM phase under the influence of DIO or not, we additionally measured the PL evolution of the pristine DPP-TT-T with and without DIO (Fig. S5c and d†). It was observed very clearly that an increasing amount of DIO hindered the self-quenching mechanisms of DPP-TT-T and delayed the gelation feature in the PL signal. The solubility tests of DPP-TT-T in $o$-xylene, mesitylene and DIO at $60{ }^{\circ} \mathrm{C}$ underpinned this argument very strongly (Table S3†). DPP-TT-T had a solubility of $11 \mathrm{mg} \mathrm{mL}^{-1}$ in $o$-xylene alone and $8 \mathrm{mg} \mathrm{mL}^{-1}$ in the $o$-xylene : mesitylene mixture, since mesitylene is a nonsolvent $\left(0.2 \mathrm{mg} \mathrm{mL}^{-1}\right.$ solubility). DIO, on the other hand, is in fact a very good solvent for DPP-TT-T with $14 \mathrm{mg} \mathrm{mL}^{-1}$ solubility. Therefore, DIO increased the quality of the host solvent in a way that the gelation process is delayed for pristine DPP-TT-T.

When a good solvent, for example, chlorobenzene (CB), was taken as the host solvent instead of the $o$-xylene : mesitylene mixture, pristine DPP-TT-T without additive formed aggregates which caused a strong LS signal (Fig. S8a $\dagger$ ). This was expected since, unlike $o$-xylene, CB does not induce gelation of the polymer, and the aggregation (and/or crystallization) of the polymer is not hindered. When DIO was added to DPP-TT-T, the LS signal reduced accordingly with the increasing amount of additive. Additionally, the self-quenching mechanism of DPP-TT-T was prohibited when DIO was added to the solution (PL signal Fig. S8a $\dagger$ ). With $4 \mathrm{v} \%$ DIO, there was almost no PL quenching observable for the pristine DPP-TT-T. When DPP-TT-T : PCBM was processed from CB, the LS signal in Fig. S8b $\uparrow$ showed a similar behavior with increasing amount of DIO. The aggregation process was almost fully prohibited when DIO was present. Similarly, the PL signal in Fig. S8b $†$ showed that DIO kept the polymer soluble, causing lower aggregation/crystallization, and thus PL quenching was reduced. Although CB is expected to have better interaction with DPP-TT-T than DIO, due to the higher vapor pressure, CB dries earlier than DIO. Hence, the additive effect of delaying the microstructure formation would be still observed due to the high solubility of both components in DIO, even though the solution was processed from a good host solvent.

Moreover, this extraordinary effect of DIO on DPP-TT$\mathrm{T}$ : PCBM was additionally compared with the effect of DIO on well-known blend systems based on poly(3-hexylthiophene) (P3HT) and poly[(5,6-difluoro-2,1,3-benzothiadiazole-4,7-diyl)-alt$\left(3,3^{\prime \prime \prime}\right.$-di(2-octyldodecyl)-2,2'; $\quad 5^{\prime}, 2^{\prime \prime}, 5^{\prime \prime}, 2^{\prime \prime \prime}$-quaterthiophene-5,5diyl)] (PffBT4T-2OD), for both of which DIO is a non-solvent. ${ }^{\mathbf{1 3 , 4 6}}$ P3HT : PCBM and PffBT4T-2OD : PCBM were coated from CB and $\mathrm{CB}$ : ODCB solutions, respectively, with the same coating and drying parameters as DPP-TT-T : PCBM. For these blends, only $3 \mathrm{v} \%$ DIO was taken. Although the drying behaviors and microstructure formation mechanisms of these systems cannot be directly compared with those of DPP-TT-T : PCBM due to differences in the interaction of solution components with the selected solvent, the presence of DIO did not delay any kinetic or thermodynamic process since LS and PL data do not indicate any differences between additive-free and additive-containing samples (Fig. S6 and S7†).

We summarize our findings with the scheme presented in Fig. 8. The suboptimal solvent mixture for DPP-TT-T : PCBM induced gelation during drying, leading to edge-on oriented crystals with face-on molecular orientation relative to the donor/acceptor interface. PCBM aggregation evolved rapidly with an aggregate size of $350 \mathrm{~nm}$. The prominent domain size within the mixed phase was determined to be $56 \mathrm{~nm}$, which probably has to be considered as one of the major factors for low device performance. At the end of the host solvent evaporation, additive-free samples were organized in the following morphology: DPP-TT-T crystals + PCBM aggregates + amorphous phase. On the other hand, in the presence of DIO, the nanostructure evolution followed a completely different path due to the good solubility of both components in DIO. It has been previously reported that 'good solvent' additives induce a two-phase microstructure after the host solvent dries (polymer crystal + amorphous phase of polymer and fullerene), while 'bad solvent' additives promote a three-phase morphology (polymer crystal + additive solution of fullerene + amorphous phase). ${ }^{35}$ Nevertheless, additives, whether good or bad solvents for polymers, are still worse solvents for polymers than the host solvents, and thus promote early polymer crystallization, due to the decreased host solvent quality, allowing more time for the polymer to crystallize. ${ }^{33}$ In this work, however, gelation and crystallization of DPP-TT-T were completely suppressed due to the presence of DIO, and the aggregation of PCBM followed a much slower pace. Mesitylene being a non-solvent for DPP-TTT was likely causing the PCBM aggregation, which was further regulated by DIO under its presence. Hence the microstructure was closer to the thermodynamic equilibrium after the host solvent evaporation, forming a solid solution consisting of an amorphous phase and little or none PCBM aggregates. As the host solvent mixture has no impact on the morphology formation, we conclude that the microstructure evolution was controlled dominantly by DIO during its evaporation. After DIO evaporation, the size of the PCBM aggregates remained at 


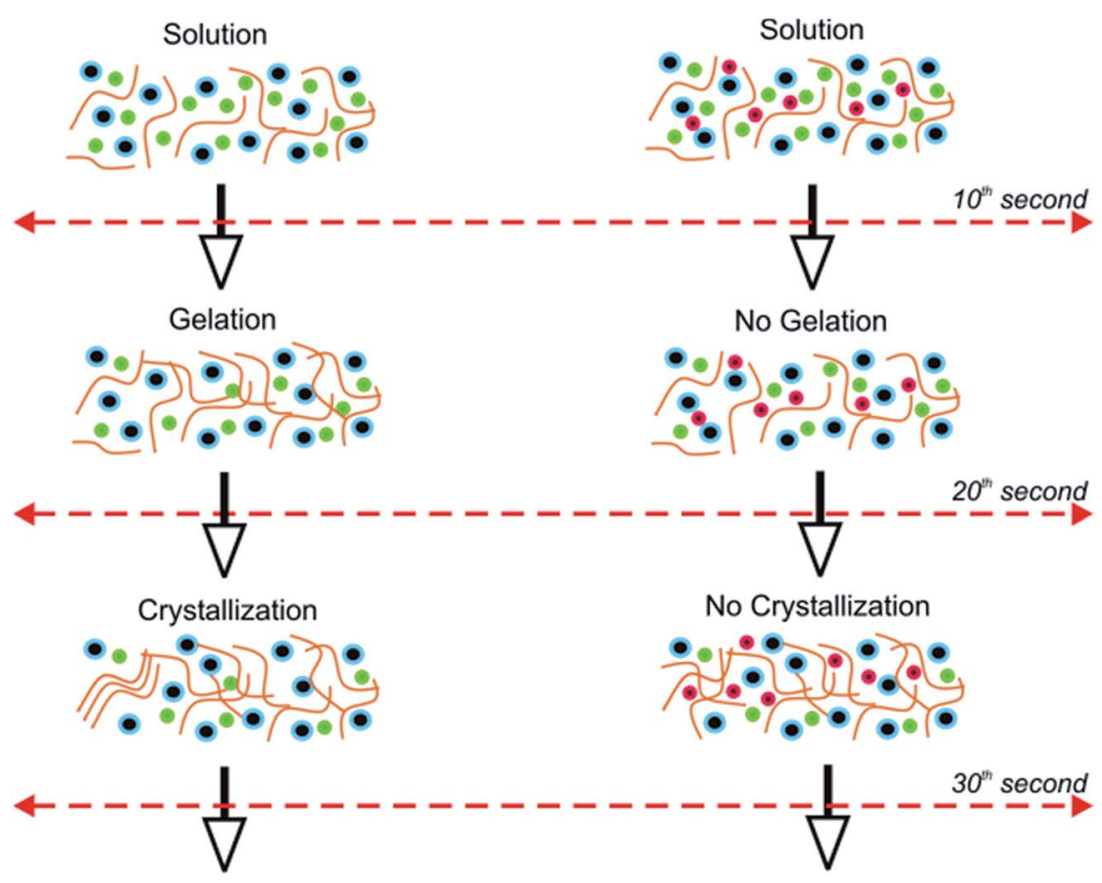

Fast PCBM aggregation

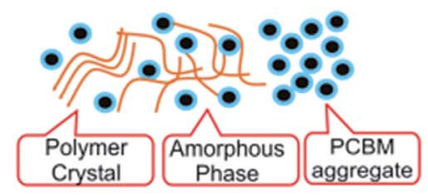

- Edge-on orientation

- Less domain purity

- Domain size $56 \mathrm{~nm}$

- PCBM aggregates $350 \mathrm{~nm}$
None or very little PCBM aggregation Mainly Amorphous Phase

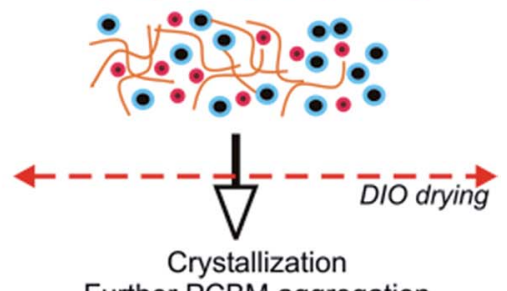

Further PCBM aggregation

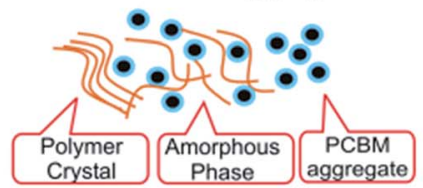

- Edge-on and face-on orientation

- Slightly more domain purity

- Domain size $29 \mathrm{~nm}$

- PCBM aggregates $100 \mathrm{~nm}$

Fig. 8 Proposed model describing film formation during the film drying process. Small blue/black circles and orange lines represent PCBM and the polymer, respectively. The red dots and green dots represent DIO and solvent mixture, respectively. The additive-free DPP-TT-T : PCBM drying (left side) experiences cross-linking of DPP-TT-T chains via gelation and crystallization, while PCBM aggregation occurs relatively fast. DIO-treated DPP-TT-T : PCBM drying (right side), on the other hand, experiences thermodynamical and kinetical delay in the morphology formation until the DIO drying.

approximately $100 \mathrm{~nm}$, while DPP-TT-T crystals adopted a bimodal orientation with a slightly more face-on molecular orientation relative to the donor/acceptor interface. The domain size at the mixed phase reduced to $29 \mathrm{~nm}$ with a slightly better domain purity, which was more desirable in terms of cell performance compared to the additive-free sample.

\section{Conclusions}

Our steady-state and in situ measurements on DPP-TT-T : PCBM reveal an alternative, previously unknown mechanism on how additives can influence the polymer : fullerene bulk-heterojunction microstructure formation. It is confirmed that the fundamental mode of operation of additives heavily depends on the host solvent quality for the polymer : fullerene bulk-heterojunctions, as the additive DIO was taken as a better solvent for both components in the DPP-TT-T : PCBM system than the host solvent mixture of $o$-xylene and mesitylene. STXM, R-SoXS and GIXD measurements indicate that DIO induces smaller PCBM aggregates as well as smaller domains but with enhanced domain purity. Face-on orientation is promoted relative to the substrate and to the donor-acceptor interface. Additionally, our in situ 
drying measurements reveal that polymer crystallization, gelation and PCBM aggregate formation are suppressed in the presence of DIO. It is confirmed that the host solvents do not play a major role in morphology evolution, and the fine nanostructure formation, especially crystallization, can be mostly 'delayed' until the drying is led solely by the additive. All in all, this work highlights that the functionality of additives and their impact on microstructure formation require more attention and more systematic investigations. This work additionally highlights that the operation mode of well-known and frequently studied additives like DIO needs to be discussed as a function of component solubility. The formation of solid solutions, where DIO keeps the polymer as well as the fullerene dissolved and unordered, is a phenomenon which requires more attention.

\section{Experimental section}

\section{Materials}

DPP-TT-T was provided by Solvay. $\mathrm{PC}_{60} \mathrm{BM}$ (99.5\%) was purchased from Solenne BV. P3HT was purchased from Merck KGaA. PffBT4T-2OD was obtained from 1-Material Inc. ZnO nanoparticle suspensions were provided by Nanograde Ltd. $\mathrm{Ba}(\mathrm{OH})_{2}$ and solvents (o-xylene (97\%), mesitylene (98\%) and 1,8diiodooctane (98\%)) were purchased from Sigma Aldrich. PEDOT : PSS (Al4083) was obtained from Heraeus. Silicon wafers, purchased from Silicon Materials with natural $\mathrm{SiO}_{2}$, were subjected to mechanical cleaning with soapy de-ionized water, rinsing with de-ionized water, ultrasonication with acetone and iso-propanol, respectively. The substrates were dried with nitrogen and kept in a desiccator to avoid any humidity effects on the surface. Solubility tests were conducted as described in detail elsewhere. ${ }^{47}$

\section{Solutions and device fabrication}

The devices were fabricated with a structure of ITO/ZnO/ $\mathrm{Ba}(\mathrm{OH})_{2} / \mathrm{DPP}-\mathrm{TT}-\mathrm{T}$ : $\mathrm{PCBM} / \mathrm{MoO}_{3} / \mathrm{Ag}$ under ambient conditions. DPP-TT-T : PCBM solutions were prepared by dissolving DPP-TT-T and PCBM in a solvent mixture with $1: 2 \mathrm{wt} \%$ and a total concentration of $15 \mathrm{mg} \mathrm{mL}^{-1}$. The solvent mixture contained $o$-xylene and mesitylene in a $7: 3$ volume ratio. Another DPP-TT-T : PCBM solution was prepared in chlorobenzene with the same ratio and concentration. The additive was added 2 vol\% with respect to the total solution volume in the glovebox atmosphere. The solutions were kept at $65{ }^{\circ} \mathrm{C}$. Pre-structured ITO-coated glass substrates were subsequently cleaned in acetone and isopropyl alcohol for $10 \mathrm{~min}$ each. After drying, the $\mathrm{ZnO}$ nanoparticle solution is doctor bladed on the glass substrates, as received. Without annealing, the $\mathrm{Ba}(\mathrm{OH})_{2}$ solution in 2-methoxyethanol ( $\left.7 \mathrm{mg} \mathrm{mL}^{-1}\right)$ was bladed on top of the $\mathrm{ZnO}$ layer and dried at $100{ }^{\circ} \mathrm{C}$ for 10 minutes. The active layer was bladed on top of the $\mathrm{Ba}(\mathrm{OH})_{2}$ layer at $60{ }^{\circ} \mathrm{C}$ with $25 \mathrm{~mm} \mathrm{~s}^{-1}$ speed, resulting in a thickness of approximately $90 \mathrm{~nm}$, as measured by using a Tencor Alphastep D-500 Profilometer. There was not any pre- or post-annealing to the cells. Finally, $15 \mathrm{~nm} \mathrm{MoO}_{3}$ and $85 \mathrm{~nm} \mathrm{Ag}$ were deposited sequentially by thermal evaporation through a shadow mask with an active area of $0.104 \mathrm{~cm}^{2} . J V$ characteristics and power conversion efficiencies of the cells were measured with a Botest source measure unit by using an Oriel Sol1A 94061 solar simulator with AM1.5G spectra of $100 \mathrm{~mW} \mathrm{~cm}^{-2}$ intensity. The P3HT : PCBM solution (1:1 wt\%) for the in situ study was prepared in chlorobenzene (CB) with a total concentration of $22.5 \mathrm{mg} \mathrm{mL}^{-1}$. The PffBT4T-2OD : PCBM solution (1:1.2 wt\%) for the in situ study was prepared in CB : ODCB (1:1 v\%) with a total concentration of $22 \mathrm{mg} \mathrm{mL}^{-1}$.

\section{Steady-state UV-Vis and photoluminescence measurements}

Absorption spectra of the films were measured on microscope slides with a Perkin Elmer Lambda 950 UV/VIS/NIR spectrophotometer. PL measurements were conducted with a custommade spectrometer equipped with a $450 \mathrm{~nm}$ laser and a Horiba Symphony InGaAs detector. PL spectra were corrected according to optical density and detector sensitivity.

\section{In situ drying measurements}

A custom-made chamber, equipped with a heat-controlled doctor blade, was used for drying measurements. The chamber with an automated solution injection system was installed on a sample stage at the European Synchrotron Radiation Facility (ESRF) at Beamline ID10 to conduct in situ drying experiments with white-light reflectometry, integrated photoluminescence and grazing-incidence X-ray diffraction (GIXD). X-ray windows (Nalophan/Kalle) were attached to the side walls of the chamber. The incident angle of the X-ray source was set to $0.16^{\circ}$ which was below the critical angle of the Si substrates. A 2D Pilatus $300 \mathrm{~K}$ detector (Dectris) was used with a frame time of $1 \mathrm{~s}$ while the sample detector distance was set to $170.33 \mathrm{~mm}$ after the calibration with silver behenate. $120 \mu \mathrm{L}$ of solution was injected onto a Si substrate, and then bladed at $60{ }^{\circ} \mathrm{C}$ with $15 \mathrm{~mm} \mathrm{~s}^{-1}$ speed under a dry helium atmosphere without any flow. All measurements were started right after the applicator passed X-ray windows and measured for 3 minutes. Any radiation damage was avoided by moving the chamber horizontally $55.5 \mu \mathrm{m}$ per frame, in total $10 \mathrm{~mm}$. Two consecutive frames had a $3.7 \mathrm{~ms}$ drying time difference, which leads to a total of $0.67 \mathrm{~s}$ drying time difference between the first and last frame of a drying experiment. Although, this is less than our time resolution, the difference was corrected between optical and GIXD measurements. White-light reflectometry (Filmetrics F20-UV) was performed with an integration time of $250 \mathrm{~ms}$. The thickness calculation method was based on interference theory as explained more in detail in the ESI. $\dagger$ Photoluminescence measurements were conducted with a $783 \mathrm{~nm}$ laser (Coherent Inc.) and an $850 \mathrm{~nm}$ long-pass filter (RG850, Schott). The signal was collected with a silicon diode (Roithner LaserTechnik $\mathrm{GmbH}$ ) and processed by a dual-phase lock-in amplifier (signal recovery). Laser light scattering measurements were conducted with the same setup under the same blading and drying conditions, together with white-light reflectometry. For this, the same measurement arrangement for PL was used with a $513 \mathrm{~nm}$ laser. There was no need for any filter since the light scattering signal was distinctly higher than the photoluminescence signal. 
P3HT : PCBM and PffBT4T-2OD : PCBM solutions were coated and dried under the same coating and drying conditions as those for DPP-TT-T : PCBM layers. Nevertheless, PL and LS measurements for these solutions were conducted with $513 \mathrm{~nm}$ and $783 \mathrm{~nm}$ lasers, respectively, due to the higher energy absorption range of $\mathrm{P} 3 \mathrm{HT}$ and PffBT4T-2OD.

\section{Ex situ morphology characterizations}

Atomic force microscopy measurements were conducted on the substrates under the same conditions as those for the device fabrication with a NT-MDT Solver Nano. Scanning transmission X-ray microscopy (STXM) and transmission electron microscopy (TEM) were performed on $\mathrm{Cu}$ grids as substrates. The samples were coated on PEDOT : PSS layers which were bladed on glass substrates from an isopropyl alcohol solution with a $1: 3$ volume ratio. The samples were cut into small pieces, floated in water, and then transferred onto a $\mathrm{Cu}$ grid. STXM experiments were performed at the PolLux end station of the Swiss Light Source (Paul Scherrer Institut, Villigen, Switzerland). ${ }^{48}$ There, zone-plate focusing was used to achieve $30 \mathrm{~nm}$ lateral resolutions in routine operation. The sample was kept at $10^{-5} \mathrm{mbar}$ vacuum and the transmitted X-ray was collected by a photomultiplier detector mounted behind the specimen. Near-edge X-ray absorption fine structure (NEXAFS) spectra were collected together with STXM measurements. Bright-field TEM studies were conducted with a Zeiss 912 Omega microscope operating at an accelerating voltage of $80 \mathrm{kV}$. Resonant soft X-ray scattering (R-SoXS) was conducted at beamline 11.0.1.2 of the Advanced Light Source (ALS) by floating the sections of films on silicon nitride windows. More information on the measurement protocol and data processing is presented in the main text, as well as in the ESI. $\dagger$ It is important to note that all ex situ measurements except AFM were performed under high vacuum. Special care was taken to measure the samples with residual additive immediately after vacuum initiation. Nevertheless, these samples might have been affected by the small reduction of the residual DIO content due to the vacuum initiation.

\section{Acknowledgements}

The authors gratefully thank Deutsche Forschungsgemeinschaft (DFG) in the framework of SFB 953 (Synthetic Carbon Allotropes) and Cluster of Excellence 'Engineering of Advanced Materials', Solar Technologies go Hybrid (SolTech) and Umweltfreundliche Hocheffiziente Organische Solarzellen (UOS) for financial support and Solvay for providing DPP-TT-T within the framework of the X10D EU project. The authors acknowledge the European Synchrotron Radiation Facility (ESRF) for giving access to synchrotron radiation facilities. The R-SoXS characterization and analysis by NCSU were supported by the Office of Naval Research (ONR grant N0014-15-1-2322). X. D is supported by the China Scholarship Council (CSC) and by the DFG research training group GRK 1896. M. M is funded by the Graduate School of Molecular Science (GSMS). N. S. G, T. A, A. O, N. L and C. J. B designed experiments and conducted WLR, PL, LS, UV-Vis, solubility and solar cell measurements. M. B, T. K, G. L and T. U conducted GIXD measurements. X. D, M. M and R. H. F participated in STXM, NEXAFS and BF-TEM measurements. X. J and H. A measured R-SoXS and evaluated the data.

\section{Notes and references}

1 F. C. Krebs, Sol. Energy Mater. Sol. Cells, 2009, 93, 394-412.

2 B. P. Rand, J. Genoe, P. Heremans and J. Poortmans, Prog. Photovolt. Res. Appl., 2007, 15, 659-676.

3 J. Peet, M. L. Senatore, A. J. Heeger and G. C. Bazan, Adv. Mater., 2009, 21, 1521-1527.

4 S. Alem, T.-Y. Chu, S. C. Tse, S. Wakim, J. Lu, R. Movileanu, Y. Tao, F. Bélanger, D. Désilets, S. Beaupré, M. Leclerc, S. Rodman, D. Waller and R. Gaudiana, Org. Electron., 2011, 12, 1788-1793.

5 B. R. Aïch, J. Lu, S. Beaupré, M. Leclerc and Y. Tao, Org. Electron., 2012, 13, 1736-1741.

6 S. Nam, J. Jang, H. Cha, J. Hwang, T. K. An, S. Park and C. E. Park, J. Mater. Chem., 2012, 22, 5543.

7 Y. Kim, S. A. Choulis, J. Nelson, D. D. C. Bradley, S. Cook and J. R. Durrant, Appl. Phys. Lett., 2005, 86, 1-3.

8 G. Li, Y. Yao, H. Yang, V. Shrotriya, G. Yang and Y. Yang, Adv. Funct. Mater., 2007, 17, 1636-1644.

9 J. K. Lee, W. L. Ma, C. J. Brabec, J. Yuen, J. S. Moon, J. Y. Kim, K. Lee, G. C. Bazan and A. J. Heeger, J. Am. Chem. Soc., 2010, 130, 3619-3623.

10 R. Lin, M. Wright and A. Uddin, Phys. Status Solidi A, 2013, 210, 1785-1790.

11 J. Peet, J. Y. Kim, N. E. Coates, W. L. Ma, D. Moses, A. J. Heeger and G. C. Bazan, Nat. Mater., 2007, 6, 497-500.

12 T. Y. Chu, S. Alem, S. W. Tsang, S. C. Tse, S. Wakim, J. Lu, G. Dennler, D. Waller, R. Gaudiana and Y. Tao, Appl. Phys. Lett., 2011, 98, 253301.

13 F. Machui, P. Maisch, I. Burgues-Ceballos, S. Langner, J. Krantz, T. Ameri and C. J. Brabec, ChemPhysChem, 2015, 16, 1275-1280.

14 C. H. Woo, P. M. Beaujuge, T. W. Holcombe, O. P. Lee and J. M. J. Frechet, J. Am. Chem. Soc., 2010, 132, 15547-15549.

15 C. V Hoven, X.-D. Dang, R. C. Coffin, J. Peet, T.-Q. Nguyen and G. C. Bazan, Adv. Mater., 2010, 22, E63-E66.

16 L. Ye, Y. Jing, X. Guo, H. Sun, S. Zhang, M. Zhang, L. Huo and J. Hou, J. Phys. Chem. C, 2013, 117, 14920-14928.

17 H. Zhou, Y. Zhang, J. Seifter, S. D. Collins, C. Luo, G. C. Bazan, T. Q. Nguyen and A. J. Heeger, Adv. Mater., 2013, 25, 1646-1652.

18 X. Zhu, F. Zhang, Q. An, H. Huang, Q. Sun, L. Li, F. Teng and W. Tang, Sol. Energy Mater. Sol. Cells, 2015, 132, 528-534.

19 S. Guo, B. Cao, W. Wang, J.-F. Moulin and P. MüllerBuschbaum, ACS Appl. Mater. Interfaces, 2015, 7, 4641-4649.

20 S. Guo, E. M. Herzig, A. Naumann, G. Tainter, J. Perlich and P. Müller-Buschbaum, J. Phys. Chem. B, 2014, 118, 344-350. 21 L. Ye, S. Zhang, W. Ma, B. Fan, X. Guo, Y. Huang, H. Ade and J. Hou, Adv. Mater., 2012, 24, 6335-6341.

22 Y. Gu, C. Wang and T. P. Russell, Adv. Energy Mater., 2012, 2, 683-690.

23 J. T. Rogers, K. Schmidt, M. F. Toney, E. J. Kramer and G. C. Bazan, Adv. Mater., 2011, 23, 2284-2288. 
24 A. J. Pearson, T. Wang, A. D. F. Dunbar, H. Yi, D. C. Watters, D. M. Coles, P. A. Staniec, A. Iraqi, R. A. L. Jones and D. G. Lidzey, Adv. Funct. Mater., 2014, 24, 659-667.

25 S. Y. Heriot and R. A. L. Jones, Nat. Mater., 2005, 4, 782-786. 26 M. Sanyal, B. Schmidt-Hansberg, M. F. G. Klein, A. Colsmann, C. Munuera, A. Vorobiev, U. Lemmer, W. Schabel, H. Dosch and E. Barrena, Adv. Energy Mater., 2011, 1, 363-367.

27 B. Schmidt-Hansberg, M. F. G. Klein, M. Sanyal, F. Buss, G. Q. G. De Medeiros, C. Munuera, A. Vorobiev, A. Colsmann, P. Scharfer, U. Lemmer, E. Barrena and W. Schabel, Macromolecules, 2012, 45, 7948-7955.

28 M. Campoy-Quiles, T. Ferenczi, T. Agostinelli, P. G. Etchegoin, Y. Kim, T. D. Anthopoulos, P. N. Stavrinou, D. D. C. Bradley and J. Nelson, Nat. Mater., 2008, 7, 158-164.

29 K. W. Chou, B. Yan, R. Li, E. Q. Li, K. Zhao, D. H. Anjum, S. Alvarez, R. Gassaway, A. Biocca, S. T. Thoroddsen, A. Hexemer and A. Amassian, Adv. Mater., 2013, 25, 19231929.

30 J. Bergqvist, S. A. Mauger, K. Tvingstedt, H. Arwin and O. Inganäs, Sol. Energy Mater. Sol. Cells, 2013, 114, 89-98.

31 L. H. Rossander, N. K. Zawacka, H. F. Dam, F. C. Krebs and J. W. Andreasen, AIP Adv., 2014, 4, 87105.

32 J. T. Rogers, K. Schmidt, M. F. Toney, G. C. Bazan and E. J. Kramer, J. Am. Chem. Soc., 2012, 134, 2884-2887.

33 N. Shin, L. J. Richter, A. A. Herzing, R. J. Kline and D. M. DeLongchamp, Adv. Energy Mater., 2013, 3, 938-948.

34 F. Liu, W. Zhao, J. R. Tumbleston, C. Wang, Y. Gu, D. Wang, A. L. Briseno, H. Ade and T. P. Russell, Adv. Energy Mater., 2014, 4, 1-9.

35 L. J. Richter, D. M. Delongchamp, F. A. Bokel, S. Engmann, K. W. Chou, A. Amassian, E. Schaible and A. Hexemer, Adv. Energy Mater., 2015, 5, 140975.
36 J. J. van Franeker, M. Turbiez, W. Li, M. M. Wienk and R. A. J. Janssen, Nat. Commun., 2015, 6, 6229.

37 Y. W. Su, C. M. Liu, J. M. Jiang, C. S. Tsao, H. C. Cha, U. S. Jeng, H. L. Chen and K. H. Wei, J. Phys. Chem. C, 2015, 119, 3408-3417.

38 N. S. Güldal, T. Kassar, M. Berlinghof, T. Ameri, A. Osvet, R. Pacios, G. Li Destri, T. Unruh and C. J. Brabec, J. Mater. Chem. C, 2016, 16-19.

39 B. J. Tremolet de Villers, K. A. O'Hara, D. P. Ostrowski, P. H. Biddle, S. E. Shaheen, M. L. Chabinyc, D. C. Olson and N. Kopidakis, Chem. Mater., 2016, 28, 876-884.

40 J. Shin, G. E. Park, D. H. Lee, H. A. Um, T. W. Lee, M. J. Cho and D. H. Choi, ACS Appl. Mater. Interfaces, 2015, 7, 32803288.

41 O. Synooka, K. R. Eberhardt, C. R. Singh, F. Hermann, G. Ecke, B. Ecker, E. Von Hauff, G. Gobsch and H. Hoppe, Adv. Energy Mater., 2014, 4, 2-11.

42 B. A. Collins, Z. Li, J. R. Tumbleston, E. Gann, C. R. Mcneill and H. Ade, Adv. Energy Mater., 2013, 3, 65-74.

43 N. D. Treat and M. L. Chabinyc, Annu. Rev. Phys. Chem., 2014, 65, 59-81.

44 J. R. Tumbleston, B. A. Collins, L. Yang, A. C. Stuart, E. Gann, W. Ma, W. You and H. Ade, Nat. Photonics, 2014, 8, 385-391.

45 B. A. Collins, J. E. Cochran, H. Yan, E. Gann, C. Hub, R. Fink, C. Wang, T. Schuettfort, C. R. McNeill, M. L. Chabinyc and H. Ade, Nat. Mater., 2012, 11, 536-543.

46 J. Zhao, S. Zhao, Z. Xu, B. Qiao, D. Huang, L. Zhao, Y. Li, Y. Zhu and P. Wang, ACS Appl. Mater. Interfaces, 2016, 8, 18231-18237.

47 F. Machui, S. Langner, X. Zhu, S. Abbott and C. J. Brabec, Sol. Energy Mater. Sol. Cells, 2012, 100, 138-146.

48 J. Raabe, G. Tzvetkov, U. Flechsig, M. Böge, A. Jaggi, B. Sarafimov, M. G. C. Vernooij, T. Huthwelker, H. Ade, D. Kilcoyne, T. Tyliszczak, R. H. Fink and C. Quitmann, Rev. Sci. Instrum., 2008, 79, 113704. 